\title{
Functionalization of carbon nanotubes with proteins and quantum dots in aqueous buffer solutions
}

\author{
R. Prakash \\ Department of Physics and Astronomy, University of North Carolina, Chapel Hill, North Carolina 27599 \\ Rich Superfine \\ Department of Physics and Astronomy, Curriculum for Applied and Materials Science, Department of \\ Computer Science, University of North Carolina, Chapel Hill, North Carolina 27599 \\ S. Washburn \\ Department of Physics and Astronomy, Curriculum for Applied and Materials Science, Department of \\ Computer Science, Biomedical Engineering, University of North Carolina, Chapel Hill, North Carolina 27599 \\ Michael R. Falvo ${ }^{\text {a) }}$ \\ Curriculum for Applied and Materials Science, Department of Physics and Astronomy University of North \\ Carolina, Chapel Hill, North Carolina 27599
}

(Received 8 May 2005; accepted 4 January 2006; published online 6 February 2006)

\begin{abstract}
We report here on a method of suspending carbon nanotubes (CNTs) in aqueous buffer solutions and functionalizing CNTs with a molecule that is "sticky" to proteins. The specific bifunctional molecule used in this study is 1-pyrene butanoic acid succidymidyl ester (1-pbase). We report successful protein and quantum dot functionalization of the CNTs, using 1-pbase as a linking agent. (C) 2006 American Institute of Physics. [DOI: 10.1063/1.2171802]
\end{abstract}

The uses of individual carbon nanotubes (CNTs) for the sensing of proteins, ${ }^{1}$ visualization, manipulation of biomolecules, and other applications are now in their nascent stages. One limiting factor for all of these applications is the ability to suspend CNTs in biologically relevant aqueous environments. There have been quite a few efforts to incorporate CNTs into such buffers including both covalent and noncovalent modifications of the inherently hydrophobic sidewall of the CNTs. ${ }^{2}$ Direct visualization of CNTs with florescence microscopy has been accomplished by exposing the hydrophobic sidewalls of CNTs with long chained aliphatic fluorescent molecules. ${ }^{3}$ There also have been reports of functionalization of substrate bound CNT, ${ }^{1,4,5}$ which avoids some of the incompatibility problems between CNTs and aqueous buffers.

In this letter, we discuss a method of functionalizing multiwalled CNTs (MWCNTs) with proteins in solution. Building on prior findings, ${ }^{6}$ we show that the functionalization of MWCNTs with 1-pyrene butanoic acid succidymidyl ester (1-pbase) enables a stable suspension of CNTs in a variety of aqueous buffers. Further, we report functionalization of MWCNTs with proteins in solution as a result of this sidewall modification. We also present the use of this molecule as a method of visualizing MWCNTs in biologically relevant environments as well as through use of fluorescent quantum dots (QDs). ${ }^{7}$

Because of the inherent hydrophobicity of CNTs $\left(s p^{2}\right.$-bonded graphene sheet), they are not soluble in aqueous buffers. Through noncovalent functionalization with 1-pbase, the MWCNT are stably suspended for a period on the order of weeks. The interaction of the 1-pbase and the MWCNT sidewall, as described by Chen, is hypothesized to be via a $\pi$ - $\pi$ bonding interaction between the pyrene of the 1-pbase and the six-membered carbon rings of the MWCNT. ${ }^{6}$ The

${ }^{a)}$ Electronic mail: falvo@physics.unc.edu
MWCNT were observed to undergo a phase transfer from the organic layer (DCE) to the aqueous layer with the 1-pbase being the phase transfer catalyst. Functionalization of MWCNT with 1-pbase and subsequent suspension in aqueous buffer was accomplished using two different methods. Method 1: 1-2 mg of MWCNT soot produced by arcdischarge (Alpha Aeser) was dispersed in DCE and then the solvent was removed with vacuum. The average MWCNT length was $2-7 \mu \mathrm{m}$ and $10-25 \mathrm{~nm}$ in width. The MWCNTs were then incubated in their original vial with $10 \mu \mathrm{l}$ of $0.5 \mathrm{mg} / \mathrm{ml}$ solution of 1-pbase in DCE for $30 \mathrm{~min}$. The excess DCE was evaporated under vacuum and the $5 \mathrm{ml}$ of buffer solution of interest was added to the vial. The vial was sonicated for 2-3 hr to ensure dispersion of the MWCNTs in suspension. Method 2:200 $\mu \mathrm{l}$ MWCNT in DCE were put into a centrifuge tube. $1 \mu \mathrm{l}$ of $0.05 \mathrm{mg} / \mathrm{ml} 1$-pbase in DCE was added to the sample. This was incubated at room temperature for 5-10 min. $200 \mu \mathrm{l}$ of the buffer solution of interest was then added to the centrifuge tube. This tube was then vortexed for $15 \mathrm{~s}$ and centrifuged to separate the DCE and aqueous layers. Then the DCE layer was removed. Optical characterization of both methods showed no measurable difference in the dispersion or concentration of MWCNT.

The pyrene ring structure of 1-pbase interacts with the sidewall of the MWCNT, thus leaving only the polar succidymidyl ester group to interact with the aqueous solution (Fig. 1), thereby solubilizning the whole complex (MWCNT/ 1-pbase). Figure 2 shows the result of the phase transfer of MWCNT from the organic to aqueous layer. Without 1-pbase, the phase transfer of MWCNT does not occur.

Further investigation of 1-pbase binding was done with atomic force microscopy (AFM). Before and after 1-pbase functionalization data show a height difference of 2-3 nm. AFM imaging was done with a Topometrix Explorer. Imaging of the 1-pbase/MWCNT was done in air using $70 \mathrm{kHz}$ silicon nitride cantilevers from Nanosensors Corp. Samples were prepared by spin coating MWCNT onto glass slides 


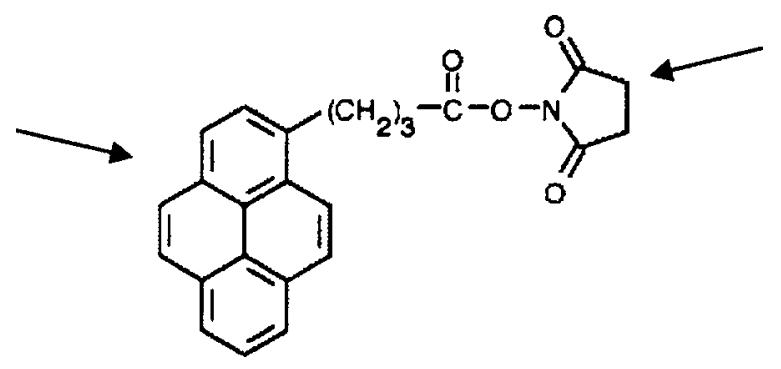

FIG. 1. Structure of 1-pyrenebutanoic acid, succinimidyl ester. The arrow on the left points to the pyrene group hypothesized to interact with the surface of the CNT. The arrow on the right points to the succinimydyl ester group which forms covalent bonds to the protein.

with $\mathrm{Au}$ fiducials in order to revisit the same tubes after modification. $10 \mu \mathrm{l}$ of $0.5 \mathrm{mg} / \mathrm{ml} 1$-pbase in DCE were put onto to the sample for $2 \mathrm{~min}$. This was then washed with DCE and nitrogen dried three subsequent times to remove excess 1-pbase. An analysis of the roughness on the MWCNT implied a fairly uniform binding of the 1-pbase on the MWCNT.

Further characterization of the composite material was done with fluorescence microscopy (1-pbase is commonly used as an amine reactive dye molecule). In Fig. 3, MWCNT are visible with both phase contrast and fluorescence imaging. This-sample was prepared using method 2 and then spin coated onto a glass slide at $2500 \mathrm{rpm}$ in four $50 \mu \mathrm{l}$ aliquots. In addition to the MWCNT, labeled carbonaceous debris can be seen (such debris is typical in MWCNT suspensions). Images were taken using Nikon inverted Diaphot 200 with Spot 2 cooled-Charge coupled device camera. Fluorescence images of the 1-pbase functionalized MWCNT were taken with an UV filter (320 excitation/380 emission) from Chroma Technology Corporation.

As reported by Chen et al., ${ }^{6}$ the use of 1-pbase allows for the immobilization of proteins on the sidewalls of CNTs. In this letter we expand this finding by specifically functionalizing MWCNT with proteins in aqueous buffer rather than on substrate bound CNTs. The protein used for this study was streptavidin, which was chosen because of the versatility of the streptavidin-biotin complex. MWCNT/streptavidin composites were prepared by mixing $1 \mu \mathrm{l}$ of $1 \mu \mathrm{g} / 100 \mu \mathrm{l}$ of streptavidin in polybutene sulfone (PBS) buffer into $200 \mu \mathrm{l}$ of the 1-pbase functionalized MWCNT in PBS solution. This

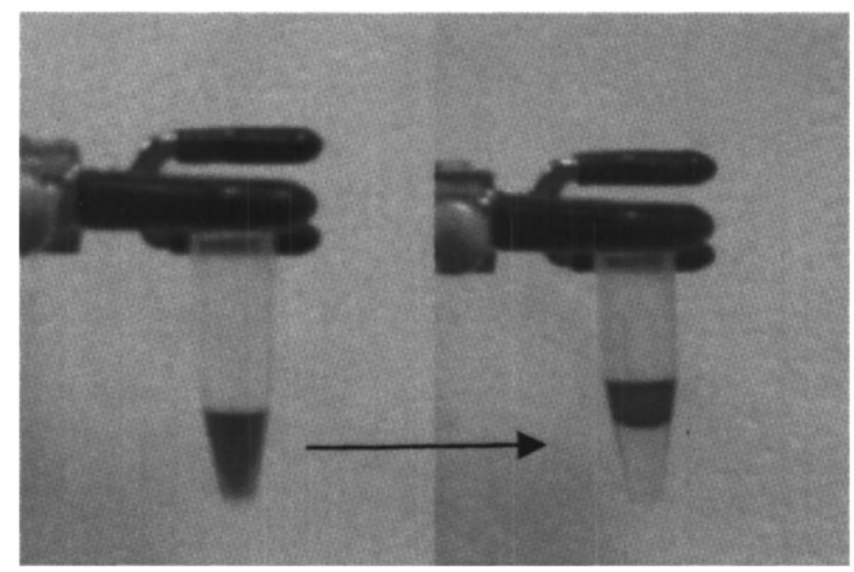

FIG. 2. MWCNT functionalized with 1-pbase in DCE (left). On the right, the top layer is the water and the bottom layer is the DCE. Arrow indicates DCE layer transition.

FIG. 4. AFM image of streptavidin on CNTs in aqueous buffer.
Downloaded 24 Apr 2006 to 152.2.131.66. Redistribution subject to AIP license or copyright, see http://apl.aip.org/apl/copyright.jsp

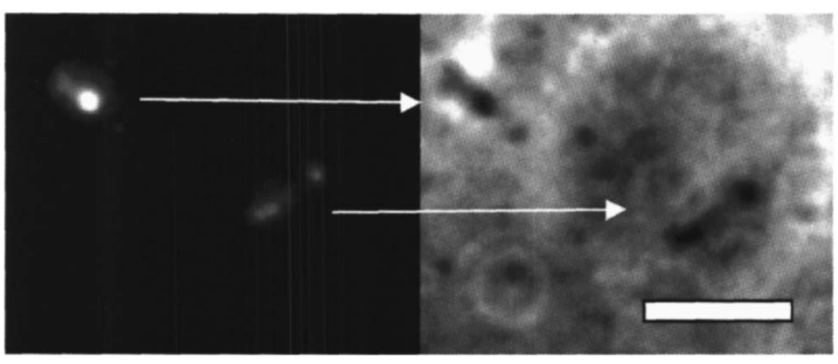

FIG. 3. Phase contrast image (left) and fluorescence image (right) of CNTs labeled with 1-pbase. Scale bars are $5 \mu \mathrm{m}$ in length.

solution was incubated at room temperature for $1 \mathrm{~h}$ before use/visualization.

Figure 4 is an AFM image taken in buffer solution, which shows streptavidin adsorption onto the sidewalls of MWCNT. The imaging substrates were glass coverslips functionalized with BSA $(10 \mathrm{mg} / \mathrm{ml})$ applied for $5 \mathrm{~min}$. BSA was added to avoid nonspecific binding of excess streptavidin in PBS onto the glass surface. The streptavidin coated MWCNT were then spin coated at $2500 \mathrm{rpm}$ onto the BSA treated glass cover slide. Images were taken in aqueous conditions under phosphate buffered silane buffer. As can be seen in Fig. 4, there is an uneven buildup of protein along the length of the MWCNT. Here the modifications were done only in solution and were untouched after being deposited onto the substrate, unlike in other studies where postprotein attachment washes were performed. In most cases, visualization was carried out using rhodamine-labeled streptavidin. Of course the fluorescence image does not have enough resolution to indicate this uneven buildup.

There has been only one other claim of functionalization of CNT with proteins in solution ${ }^{5}$ (as opposed to substrate bound CNT studies). In that study, the CNT sidewall was modified covalently, altering the unique electronic properties of the CNT. The attachment of 1-pbase as alluded to by Chen et al. is noncovalent in nature and thus should not disrupt the electrical properties of the CNTs. Several studies report on the effect of conjugated ring structures adsorbed onto CNTs, ${ }^{9}$ which show a change in the resistivity of the SWCNT, but not enough to alter the electronic properties significantly.

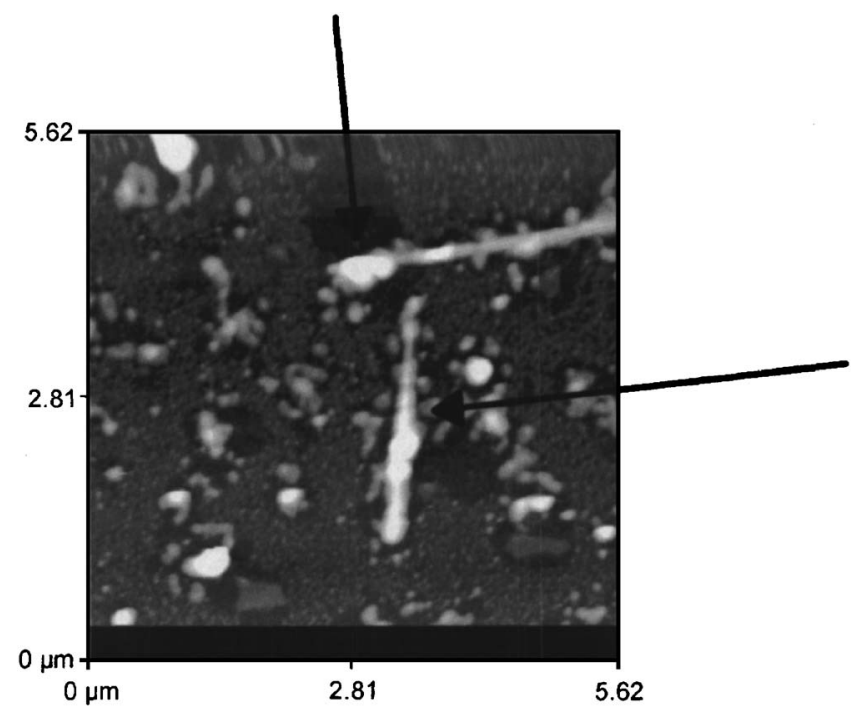




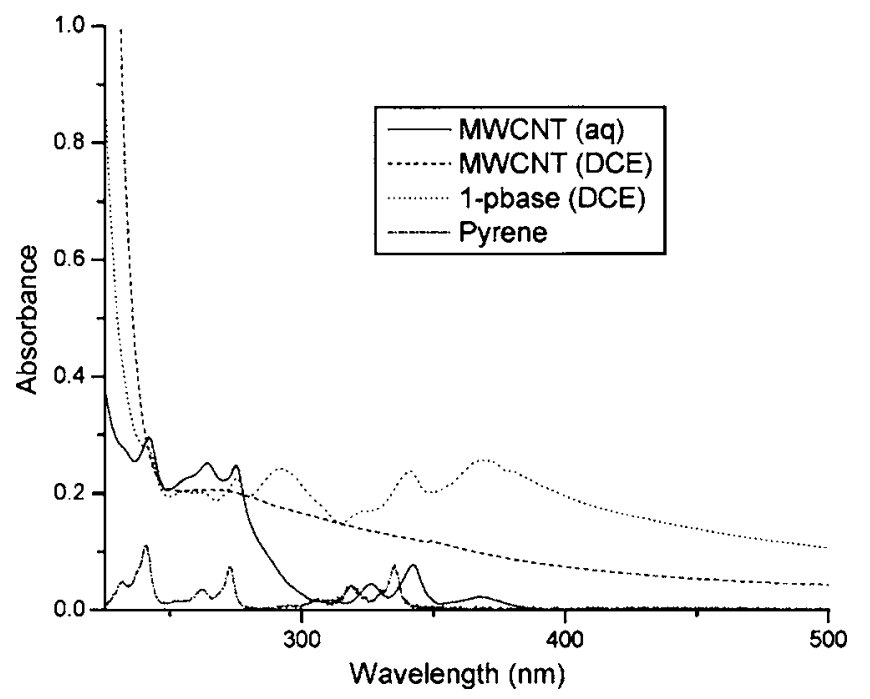

FIG. 5. Ultraviolet-visible spectra showing the electronic change of the MWCNT due to the adsorption of 1-pbase onto CNTs.

UV-visible spectra data can elucidate the interaction between the CNT and the 1-pbase (see Fig. 5). UV-visible data taken with a Varian Cary 400 Bio UV-visible spectrophotometer. Samples of MWCNT in DCE, 1-pbase in DCE, and 1-pbase/MWCNT composites in water [MWCNT (aq)] were all taken at room temperature with the appropriate blanks to eliminate solvent absorption. For reference, the catalogued spectra of pyrene in cyclohexanol is also shown in this plot. ${ }^{10} \mathrm{We}$ see that there is only one peak from the 1-pbase spectrum absent in the MWCNT (aq) spectrum at approximately $275 \mathrm{~nm}$. This missing peak should be in a place of increasing absorption for the MWCNT (aq), whose increase could mask the peak. We note because the 1-pbase is insoluble in aqueous solutions, the absorption of light in MWCNT (aq) is coming primarily from the functionalized MWCNTs and not from the possible excess 1-pbase in the sample. Looking at the pyrene spectra we see that all of its peaks are present in the MWCNT (aq) spectra, but are redshifted. We attribute this shift to solvent affects as described by Waters ${ }^{11}$ and others. We note that this noncovalent interaction with the MWCNT would not dramatically change the MWCNT's electronic properties.

We have also used a similar protocol to functionalize CNT with QD. $1 \mu \mathrm{l} 2 \mu \mathrm{M}$ QD 605 streptavidin conjugates (Quantum Dot Corp.) was diluted 1:10 in PBS buffer. $1 \mu \mathrm{l}$ of this was added to $199 \mu \mathrm{l}$ of the 1-pbase/MWCNT suspension in PBS buffer. This was allowed to incubate at room

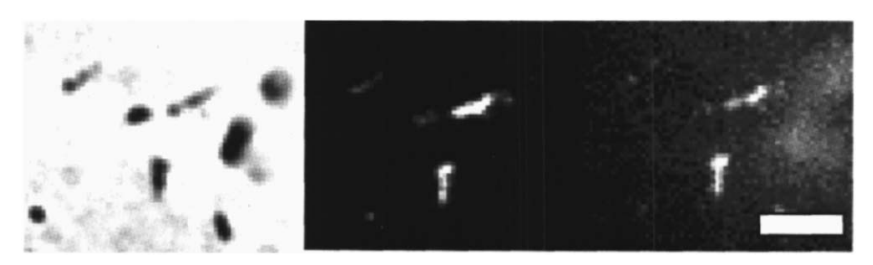

FIG. 6. This series of images shows the functionalization of MWCNTs with streptavidin coated QDs and then subsequent functionalization with biotin. The scale bars in all of these images are $2.5 \mu \mathrm{m}$ in length. temperature for $30 \mathrm{~min}$. Figure 6 shows single frame capture of the QD on the MWCNT. There has been only one other report of the attachment of QD to CNT through noncovalent specific binding. ${ }^{12}$

We then investigated the chemical activity of the streptavidin on the conjugated QD with biocitin-488, which has a strong affinity for streptavidin. Biotin, labeled with Alexaflour 488 (Molecular Probes) was diluted to $0.1 \mathrm{mg} / \mathrm{ml} .1 \mu \mathrm{l}$ of this was added to the MWCNT/QD solution and allowed to incubate for $30 \mathrm{~min}$ at room temperature. In Fig. 6, the biotin is clearly visible on the CNT. This implies that the QD/CNT complex can be functionalized easily in solution with any protein that has been biotintylated. In theory, this result is quite surprising as the ratio of QD to streptavidin is $1: 1$. Because there are multiple binding sites for biotin to streptavidin, we still see relatively uniform binding across the CNT. Images of the QDs functionalized MWCNT were taken using a TRITC filter, and images of biocitin on MWCNT were taken using a FITC filter. We note that the QDs were not visible with fluorescence using the FITC filter and the biotin was not visible using the TRITC filter; this allowed us to distinguish the two materials.

In this letter we show a new way of functionalizing CNTs with protein in aqueous buffer solutions. We have found that noncovalent functionalization of CNT with 1-pbase allows us to stably solubilize the CNTs and to perform successful protein absorption in solution. This work will inform future studies of CNT/protein complexes in applications that require them to be dissolved in aqueous environments.

The authors acknowledge support from NSF and L.E. McNeil for her helpful insights on UV Spectroscopy. They also acknowledge valuable conversations with M. L. Waters.

${ }^{1}$ K. Besteman, J. O. Lee, F. G. M. Wiertz, H. A. Heering, and C. Dekker, Nano Lett. 3, 727 (2003); K. Bradley, M. Briman, A. Star, and G. Gruner, ibid. 4, 253 (2004); R. J. Chen, S. Bangsaruntip, K. A. Drouvalakis, N. W. Kam, M. Shim, Y. Li, W. Kim, P. J. Utz, and H. Dai, Proc. Natl. Acad. Sci. U.S.A. 100, 4984 (2003).

${ }^{2}$ D. Tasis, N. Tagmatarchis, V. Georgakilas, and M. Prato, Chem.-Eur. J. 9, 4001 (2003).

${ }^{3}$ R. Prakash, S. Washburn, R. Superfine, R. E. Cheney, and M. R. Falvo, Appl. Phys. Lett. 83, 1219 (2003).

${ }^{4}$ A. Star, T. R. Han, V. Joshi, J. C. P. Gabriel, and G. Gruner, Advanced Materials 16, 2049 (2004); M. Shim, N. W. S. Kam, R. J. Chen, Y. M. Li, and H. J. Dai, Nano Lett. 2, 285 (2002); C. Richard, F. Balavoine, P. Schultz, T. W. Ebbesen, and I. C. Mioskowski, Science 300, 775 (2003). ${ }^{5}$ D. Pantarotto, C. D. Partidos, R. Graff, J. Hoebeke, J. P. Briand, M. Prato, and A. Bianco, J. Am. Chem. Soc. 125, 6160 (2003).

${ }^{6}$ R. J. Chen, Y. G. Zhan, D. W. Wang, and H. Dai, J. Am. Chem. Soc. 123, 3838 (2001).

${ }^{7}$ X. Michalet, F. Pinaud, T. D. Lacoste, M. Dahan, M. P. Bruchez, A. P. Alivisatos, and S. Weiss, Single Mol. 2, 261 (2001).

${ }^{8}$ S.-P. Han, T. Cagin, and W. A Goddard III, presented at the Material Research Society Symposium Proceedings, 2003 (unpublished).

${ }^{9}$ A. Star, T. R. Han, J. C. P. Gabriel, K. Bradley, and G. Gruner, Nano Lett. 3, 1421 (2003); J. J. Zhao, J. P. Lu, J. Han, and C. K. Yang, Appl. Phys. Lett. 82, 3746 (2003).

${ }^{10}$ H. Du, R. C. A. Fuh, J. Z. Li, A. Corkan, and J. S. Lindsey, Photochem. Photobiol. 68, 141 (1998).

${ }^{11}$ M. L. Waters, Curr. Opin. Chem. Biol. 6, 736 (2002).

${ }^{12}$ S. Ravindran, S. Chaudhary, B. Colburn, M. Ozkan, and C. S. Ozkan, Nano Lett. 3, 447 (2003). 